\title{
Synthesis of some aryl (pyrimidin-3-yl)methyl ureas, hydrazones and semicarbazones
}

\author{
Virginija Jakubkiene,* Justinas Zurba, and Milda M. Burbuliene \\ Department of Organic Chemistry, Faculty of Chemistry, Vilnius University \\ Naugarduko 24, LT-03225, Vilnius, Lithuania \\ E-mail:virginija.jakubkiene@,chf.vu.lt
}

DOI: $\underline{\text { http://dx.doi.org/10.3998/ark.5550190.p009.620 }}$

\begin{abstract}
Two methods of synthesis of aryl (pyrimidin-3-yl)methyl ureas were investigated. One of them involved reaction sequence starting with (pyrimidin-3-yl)acetohydrazides to give the corresponding azides, which reacted with aryl amines to form aryl (pyrimidin-3-yl)methyl ureas. The second one was based on a one-pot reaction of (pyrimidin-3-yl)acetic acids with diphenyl phosphoryl azide followed by reaction with aryl amines. Also, some new aryl and (pyrimidin-3yl) moiety bearing hydrazones and semicarbazones were synthesized.
\end{abstract}

Keywords: (Pyrimidin-3-yl)acetyl azides, aryl (pyrimidin-3-yl)ureas, hydrazones, semicarbazones

\section{Introduction}

Pyrimidines represent an important group of heterocyclic compounds exhibiting broad spectrum of biological activity. ${ }^{1-5}$ Moreover, pyrimidine moiety is a building block for some new drug introduced to the market every year (Figure 1). Thus, dabrafenib mesylate (Tafinlar ${ }^{\mathbb{B}}$ ) was approved in 2013 for the treatment of metastatic BRAF-mutant melanoma, macitentan $\left(\right.$ Opsumit $^{\circledR}$ ) and riociguat (Adempas ${ }^{\circledR}$ ) - for the treatment of pulmonary arterial hypertension (PAH), sofosbuvir (Sovaldi ${ }^{\circledR}$ ) - for the treatment of the hepatits $\mathrm{C}$ virus (HCV) across several genotypes. ${ }^{6}$ On the other hand, compounds with the moiety of urea, hydrazone and semicarbazone are citied to show anticancer, ${ }^{7-9}$ antimicrobial, ${ }^{1,3-5,10-13}$ antithrombotic, ${ }^{14}$ analgesic $^{15,16}$ and anti-inflammatory activity. $1,5,13,16$ 
<smiles>CC(C)(C)c1nc(-c2cccc(NS(=O)(=O)c3c(F)cccc3F)c2F)c(-c2ccnc(N)n2)s1</smiles>

Dabrafenib mesylate<smiles>COC(=O)N(C)c1c(N)nc(-c2nn(Cc3ccccc3F)c3ncccc23)nc1N</smiles>

Riociguat<smiles>CCCNS(=O)(=O)Nc1ncnc(OCCOc2ncc(Br)cn2)c1-c1ccc(Br)cc1</smiles>

Macitentan<smiles>CC(C)OC(=O)C(C)CP(=O)(O)OCC1O[C@@H](n2ccc(=O)[nH]c2=O)[C@](C)(F)[C@H]1O</smiles>

Sofosbuvir

Figure 1. New drugs having a pyrimidine skeleton.

Earlier we have synthesised arylpyrimidinylureas and carbamates, which showed antiinflammatory activity against endotoxin-induced airway epithelial cell injury ${ }^{17,18}$ (Scheme 1).<smiles>[R]c1ccc(NC(=O)NCn2c(=O)cc(C)[nH]c2=O)c([R])c1[R]</smiles>

4-9<smiles>[R]c1ccc(OC(=O)NCn2c(=O)cc(C)[nH]c2=O)cc1</smiles>

2, $\mathrm{R}=\mathrm{H}$

$3, \mathrm{R}=\mathrm{Cl}$

$$
\begin{aligned}
& \mathrm{R}^{1}=\mathrm{R}^{2}=\mathrm{R}^{3}=\mathrm{H}(\mathbf{4}) ; \\
& \mathrm{R}^{1}=\mathrm{CH}_{3}, \mathrm{R}^{2}=\mathrm{R}^{3}=\mathrm{H}(\mathbf{5}) ; \\
& \mathrm{R}^{1}=\mathrm{R}^{2}=\mathrm{H}, \mathrm{R}^{3}=\mathrm{OCH}_{3}(\mathbf{6}) ; \\
& \mathrm{R}^{1}=\mathrm{R}^{2}=\mathrm{H}, \mathrm{R}^{3}=\mathrm{F}(\mathbf{7}) ; \\
& \mathrm{R}^{1}=\mathrm{R}^{3}=\mathrm{Cl}, \mathrm{R}^{2}=\mathrm{H}(\mathbf{8}) \\
& \mathrm{R}^{1}=\mathrm{R}^{3}=\mathrm{H}, \mathrm{R}^{2}=\mathrm{CF}_{3}(\mathbf{9})
\end{aligned}
$$

Scheme 1. Reagents and conditions: (i) corresp. phenol, dry dioxane, reflux, $5 \mathrm{~h}$; (ii) corresp. aniline, dry dioxane, reflux, $1 \mathrm{~h}$. 
$N$-(2,4-Dichlorophenyl)- $N$ '-[(6-methyl-2,4-dioxo-1,2-dihydropyrimidin-3(4H)-

yl)methyl]urea (8) showed especially high activity compared to that of emodin and rhein. ${ }^{18}$ On the basis of the above mentioned structures, bearing urea bridged 2,4-dioxopyrimidine and halo aryl fragment, the goal of our reasearch was to synthesize series of new structurally relative compounds as well as to find out the most effective methods for their synthesis.

\section{Results and Discussion}

The synthesis of the starting compounds $\mathbf{1 2}, \mathbf{1 3}$ and $\mathbf{1 5}$ is described in Scheme 2. Thus, compound $\mathbf{1 1}$ was synthesized by bromination of ester $\mathbf{1 0}$ according to the known procedure. ${ }^{19}$ Compound 15 was synthesized by analogous bromination from hydrazide $\mathbf{1 4}$ which synthesis has already been described. ${ }^{20}$ Hydrolysis of esters $\mathbf{1 0}, \mathbf{1 1}$ with $20 \% \mathrm{NaOH}$ solution, then acidification with conc. $\mathrm{HCl}$ gave the corresponding acids 12, 13. Acids 12, 13 and hydrazides 14,15 were further used for acyl azide 1, 16 synthesis.<smiles>[R]c1c(C)nc(SC)n(CC(=O)OCC)c1=O</smiles><smiles>[R]c1c(C)[nH]c(=O)n(CC(=O)O)c1=O</smiles>

$\mathrm{i} \longrightarrow \begin{array}{r}10, R \\ \longrightarrow 11, R=\mathrm{H}\end{array}$<smiles>Cc1cc(=O)n(CC(=O)NN)c(=O)[nH]1</smiles>

14
12, $\mathrm{R}=\mathrm{H}$

13, $\mathrm{R}=\mathrm{Br}$<smiles>Cc1[nH]c(=O)n(CC(=O)NN)c(=O)c1Br</smiles>

15

Scheme 2. Reagents and conditions: (i) $\mathrm{Br}_{2}, \mathrm{CH}_{3} \mathrm{COOH}$, rt, $1 \mathrm{~h}$; (ii) $20 \% \mathrm{NaOH}$, reflux, $1 \mathrm{~h}$; (iii) conc. $\mathrm{HCl}, \mathrm{pH}=2$.

Acyl azides commonly are prepared from carboxylic acids via mixed anhydrides ${ }^{21-24}$ or directly using diphenyl phosphoryl azide ${ }^{25}$ or from acyl chlorides. ${ }^{21}$ They can also been prepared by nitrosation of hydrazides. ${ }^{26,27}$

Hydrazide nitrosation was used in a previous work ${ }^{17}$ to synthesize azide 1 . In the present study, the same procedure was used for the synthesis of $\mathbf{1 6}$ which was obtained in a moderate $61 \%$ yield from hydrazide 15. According to the same nitrosation procedure from hydrazide 14, the desired azide 1 was isolated in a 73\% yield. We performed, however, one-pot two-step reaction beginning with acid $\mathbf{1 2}$ to synthesize azide $\mathbf{1}$. Treatment of acid $\mathbf{1 2}$ with 
ethylchloroformate in the presence of triethylamine followed by reaction with sodium azide, yielded only $35 \%$ of azide $\mathbf{1}$. Therefore, acyl azides $\mathbf{1}$ and $\mathbf{1 6}$ to be used for the synthesis of ureas 17-23, were prepared from corresponding hydrazides 14, 15 (Scheme 3).<smiles>[R]c1c(C)[nH]c(=O)n(CC(=O)NN)c1=O</smiles><smiles>[R]c1ccc(/C=N/NC(=O)Cn2c(=O)cc(C)[nH]c2=O)c([R])c1</smiles>

24, $\mathrm{R}^{1}=\mathrm{Cl}, \mathrm{R}^{2}=\mathrm{H}$ 25, $\mathrm{R}^{1}=\mathrm{R}^{2}=\mathrm{Cl}$<smiles>[R]c1c(C)[nH]c(=O)n(CC(=O)O)c1=O</smiles>

$$
\text { 12, } \mathrm{R}=\mathrm{H}
$$

13, $\mathrm{R}=\mathrm{Br}$<smiles>C=[Te][V]</smiles><smiles>[R]c1c(C)[nH]c(=O)n(CC(N)=O)c1=O</smiles>

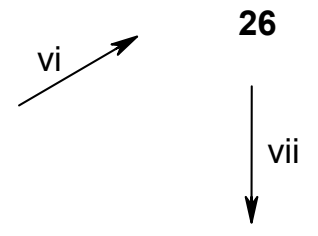<smiles>[R][X]c1ccc(/C=N/NC(=O)NCn2c(=O)cc(C)[nH]c2=O)c([R])c1</smiles><smiles>[R]</smiles>
16, $\mathrm{R}=\mathrm{Br}$<smiles>Cc1cc(=O)n(CNC(=O)NN)c(=O)[nH]1</smiles>

26

$$
\begin{aligned}
& \text { 27, } R^{1}=\mathrm{Cl}, \mathrm{R}^{2}=\mathrm{H} \\
& \text { 28, } \mathrm{R}^{1}=\mathrm{R}^{2}=\mathrm{Cl}
\end{aligned}
$$<smiles>[R]c1cc([R])c(NC(=O)NCn2c(=O)[nH]c(C)c([R])c2=O)c([R])c1</smiles>

8, 17-23

$$
\begin{aligned}
& R=R^{1}=H, R^{2}=R^{3}=C l(8) ; R=R^{1}=R^{2}=H, R^{3}=C l(17) ; R=R^{1}=R^{3}=H, R^{2}=C l(18) ; \\
& R=R^{2}=H, R^{1}=R^{3}=C l(19) ; R=R^{1}=R^{3}=H, R^{2}=B r(20) ; R=R^{1}=H, R^{2}=R^{3}=B r(21) ; \\
& R=B r, R^{1}=H, R^{2}=R^{3}=C l(22) ; R=R^{1}=R^{2}=B r, R^{3}=H(23) .
\end{aligned}
$$

Scheme 3. Reagents and conditions: (i) aq. $\mathrm{HCl}, \mathrm{NaNO}_{2} / \mathrm{H}_{2} \mathrm{O}, 0-5{ }^{\circ} \mathrm{C}, 30 \mathrm{~min}$.; (ii) abs. acetone, TEA, $\mathrm{ClCO}_{2} \mathrm{Et},-5-5{ }^{\circ} \mathrm{C}, 30 \mathrm{~min}$., then $\mathrm{NaN}_{3} / \mathrm{H}_{2} \mathrm{O}, 0-5{ }^{\circ} \mathrm{C}, 7 \mathrm{~h}$, later $5-10{ }^{\circ} \mathrm{C}$ overnight - yield 1; (iii) dry benzene, TEA, rt, 10 min., then DPPA, rt, 24 h: (iv) dry benzene, corresp. aniline, reflux, $3 \mathrm{~h}$; (v) $i$-PrOH, reflux, $3 \mathrm{~min}$., then $70-75^{\circ} \mathrm{C}$, corresp. benzaldehyde; (vi) dry benzene, reflux, $2 \mathrm{~h}$, then diethyl ether, $\mathrm{N}_{2} \mathrm{H}_{4} \cdot \mathrm{H}_{2} \mathrm{O}$, rt, 1 h; (vii) abs. EtOH, corresp. benzaldehyde, rt, $24 \mathrm{~h}$.

Acyl azides undergo thermal rearrangement in an inert solvents to give isocyanates (Curtius rearrangement), which on reaction with amines lead to the formation of ureas. ${ }^{22,25,27}$ Thus, heating at reflux acyl azides 1, 16 and arylamines in dry benzene produced ureas 8, 17-23 in 53- 
$76 \%$ yields. The structure of these compounds was confirmed by NMR data. The number of signals and their chemical shifts are in accordance with the given structures. For example, in the ${ }^{1} \mathrm{H}$ NMR spectra of ureas 8, 17-23 the characteristic splitting of $\mathrm{NCH}_{2}$ group protons into doublets (5.20-5.28 ppm) and neighbour NH protons triplets (6.90-7.93 ppm) are observed. The $\mathrm{NH}$ protons close to aromatics resonate at $8.25-8.90 \mathrm{ppm}$.

It should be noted, that the yield and the reaction time are dependent on the number of halogen atoms and their position in an aryl amine ring, e.g., azide 1 reaction with 2,6dichloroaniline occurred within $4 \mathrm{~h}$, while it needs only $2 \mathrm{~h}$ in the other cases. In addition, urea 19 is obtained in a lower yield (53\%), and 2,4,6-trichloroaniline did not react with azide $\mathbf{1}$ under given conditions.

Searching for more efficient synthesis of ureas, we tried one-pot synthetic pathway from acids 12, 13. Treatment of acids 12, 13 with diphenyl phosphoryl azide in the presence of triethylamine in dry benzene at room temperature to form acyl azides 1, 16, which without isolation from the reaction mixture further were allowed to react with corresponding anilines. According to this procedure ureas 8, 19, 21-23 were synthesized.

This synthetic pathway compared to the synthesis of ureas from hydrazides, has some advantages (less stages and shorter time), however it has disadvantages as well. First of all, to full conversion of starting acids 12, $\mathbf{1 3}$ it is necessary to use a large excess of reagents (4 equiv. of DPPA and 1 equiv. of TEA per 1 equiv. of acid). On the other hand, the output of these reactions is much lower $(8-52 \%)$. Thus, the discussed synthesis of ureas from acids would be better, presumably, only if the synthesis of the starting acids could be more convenient than synthesis of the corresponding hydrazides.

With the purpose to modify the linkage between the pyrimidine ring and aryl substituents we synthesized hydrazones 24, 25. Good yields (77 and 86\%) of hydrazones were obtained under treatment of hydrazide 14 with the corresponding benzaldehydes in hot isopropanol. In the ${ }^{1} \mathrm{H}$ NMR spectra of hydrazones 24, 25 along with others the characteristic two sets of signals of $\mathrm{NCH}_{2}$ (4.47-4.88 ppm), 6'-ArH (7.93-8.02), N=CH (8.34-8.58 ppm), CONH (11.24-11.27 ppm) and NH group of the pyrimidine ring (11.81-11.94 ppm) protons were observed. According to the literature acyl hydrazones in solutions may exist as $Z / E$ geometrical isomers about $\mathrm{C}=\mathrm{N}$ double bond and syn/anti conformers arising from the hindered rotation about amide $\mathrm{C}-\mathrm{N}$ linkage. ${ }^{28}$ In dimethyl- $d_{6}$ sulfoxide solution hydrazones are present in the form of $E$ isomers, ${ }^{29}$ so the above signals were assigned to syn/anti conformers of the $E$ geometrical isomers.

Structurally related to ureas semicarbazones also can be synthesized from acyl azides. Thus, acetyl azide 1 rearranged to isocyanate in benzene at reflux and then was allowed to react with hydrazine hydrate to form semicarbazide 26. Reaction of $\mathbf{2 6}$ with benzaldehydes yielded the corresponding semicarbazones 27, 28. In the ${ }^{1} \mathrm{H}$ NMR spectra of compounds $\mathbf{2 7}, 28$ characteristic for $\mathrm{N}=\mathrm{CH}$ proton singlets at 8.25 and $8.20 \mathrm{ppm}$ as well as signals in the area of 7.39-7.95 ppm of aromatic protons were observed. 


\section{Conclusions}

The research once again confirms that the most convenient way to synthesize (pyrimidin-3yl)acetyl azides is nitrosation of the corresponding acetohydrazides, which in turn are valuable synthons for hydrazone synthesis. Aryl (pyrimidin-3-yl)methyl ureas can be synthesized by two methods - starting with (pyrimidin-3-yl)acetyl azides or from (pyrimidin-3-yl)acetic acids. (Pyrimidin-3-yl)acetyl azides heated in dry benzene undergo rearrangement to isocyanate, which in reaction with aryl amines gave ureas. (Pyrimidin-3-yl)acetic acids reacted with diphenyl phosphoryl azide in the presence of triethylamine in dry benzene to form (pyrimidin-3-yl)acetyl azides following by in situ reaction with aryl amines to yield the corresponding ureas, i.e., additional procedure for isolation of azides was not required.

\section{Experimental Section}

General. Melting points were determined in open capillaries with a digital melting point IA9100 series apparatus (ThermoFischer Scientific) and are uncorrected. All reactions and purity of the synthesized compounds were monitored by TLC using Silica gel $60 \mathrm{~F}_{254}$ aluminium plates (Merck). Visualisation was accomplished by UV light. IR spectra were recorded on an FTIR spectrophotometer Spectrum BX II (Perkin Elmer) as potassium bromide pellets. NMR spectra were recorded on a Bruker Ascend 400 (400 and $100 \mathrm{MHz}$, respectively). ${ }^{1} \mathrm{H}$ and ${ }^{13} \mathrm{C}$ NMR were referenced to residual solvent peaks. High Resolution Mass Spectrometry (HRMS) analyses were carried out on a ESI TOF 6230 (Agilent Technologies) mass spectrometer.

General procedure for the bromination of compound 10 and 14. To a mixture of ester 10 or hydrazide $14(5 \mathrm{mmol})$ in glacial acetic acid $(7 \mathrm{~mL})$, the bromine $(0.58 \mathrm{ml}, 10 \mathrm{mmol})$ was added dropwise under stirring at room temperature. The reaction mixture was stirred at this temperature for $1 \mathrm{~h}$, the precipitate was collected by filtration, washed with water or acetone and dried over sodium sulphate in vacuum.

Ethyl-(5-bromo-6-methyl-2-methylthio-4-oxo-3,4-dihydropyrimidin-3-yl)acetate (11). White solid, yield $1.43 \mathrm{~g}(89 \%), \mathrm{mp} 128-130{ }^{\circ} \mathrm{C}$. IR, $v, \mathrm{~cm}^{-1}: 1686,1741(\mathrm{C}=\mathrm{O}) .{ }^{1} \mathrm{H}$ NMR $\left(\mathrm{CDCl}_{3}\right), \delta$, ppm: 1.31 (t, $\left.J 7.2 \mathrm{~Hz}, 3 \mathrm{H}, \mathrm{OCH}_{2} \underline{\mathrm{CH}}_{3}\right), 2.48\left(\mathrm{~s}, 3 \mathrm{H}, \mathrm{CH}_{3}\right), 2.60\left(\mathrm{~s}, 3 \mathrm{H}, \mathrm{SCH}_{3}\right), 4.27(\mathrm{k}, J 7.2 \mathrm{~Hz}$, $\left.2 \mathrm{H}, \mathrm{OCH}_{2} \mathrm{CH}_{3}\right), 4.85\left(\mathrm{~s}, 2 \mathrm{H}, \mathrm{NCH}_{2}\right) ;{ }^{13} \mathrm{C} \mathrm{NMR}\left(\mathrm{CDCl}_{3}\right), \delta$, ppm: $14.1\left(\mathrm{SCH}_{3}\right), 15.3\left(\mathrm{CH}_{3}\right), 24.8$ (6- $\left.\mathrm{CH}_{3}\right), 46.0\left(\mathrm{NCH}_{2}\right), 62.2\left(\mathrm{OCH}_{2}\right), 107.3(5-\mathrm{C}), 158.3,159.6,161.1,166.2$ (2-C, 4-C, 6-C, $\mathrm{C}=\mathrm{O}$ ). HRMS (ES): $[\mathrm{M}+\mathrm{H}]^{+}$calculated for $\mathrm{C}_{10} \mathrm{H}_{13} \mathrm{BrN}_{2} \mathrm{O}_{3} \mathrm{~S}$, 322.9883; found 322.9885 .

(5-Bromo-6-methyl-2,4-dioxo-1,2-dihydropyrimidin-3(4H)-yl)acetohydrazide (15). White solid, yield $1.05 \mathrm{~g} \mathrm{(76 \% ),} \mathrm{mp}>300^{\circ} \mathrm{C}$ (dec.). IR, $v, \mathrm{~cm}^{-1}$ : 1637, 1697, $1737(\mathrm{C}=\mathrm{O}) ; 3203,3248$ (NH). ${ }^{1} \mathrm{H}$ NMR (DMSO- $d_{6}$ ), $\delta$, ppm: $2.28\left(\mathrm{~s}, 3 \mathrm{H}, \mathrm{CH}_{3}\right), 4.03$ (br s, $\left.2 \mathrm{H}, \mathrm{NH}_{2}\right), 4.55(\mathrm{~s}, 2 \mathrm{H}$, $\left.\mathrm{NCH}_{2}\right), 11.23(\mathrm{~s}, 1 \mathrm{H}, \mathrm{NH}), 11.82(\mathrm{~s}, 1 \mathrm{H}, \mathrm{NH}) ;{ }^{13} \mathrm{C}$ NMR (DMSO- $\left.d_{6}\right), \delta, \mathrm{ppm}: 20.0\left(\mathrm{CH}_{3}\right), 42.3$ 
$\left(\mathrm{NCH}_{2}\right), 94.7$ (5-C), 150.4, 151.2, $159.4,166.9$ (2-C, 4-C, 6-C, C=O). HRMS (ES): [M+Na] calculated for $\mathrm{C}_{7} \mathrm{H}_{9} \mathrm{BrN}_{4} \mathrm{NaO}_{3}, 298.9750$; found 298.9758 .

General procedure for the synthesis of acetic acids 12 and 13. A mixture of compound $\mathbf{1 0}$ or $11(5 \mathrm{mmol})$ and solution of $20 \%$ sodium hydroxide $(3.2 \mathrm{~mL})$ was heated at reflux for $2 \mathrm{~h}$, then cooled to room temperature. The reaction mixture was acidified with conc. hydrochloric acid to $\mathrm{pH} 2$ and kept at temperature $5-10{ }^{\circ} \mathrm{C}$ for $2 \mathrm{~h}$. The resultant precipitate was collected by filtration, washed with cold water and dried.

(6-Methyl-2,4-dioxo-1,2-dihydropyrimidin-3(4H)-yl)acetic acid (12). White powder, yield $0.58 \mathrm{~g}(63 \%), \mathrm{mp} 245-246{ }^{\circ} \mathrm{C}$; (mp $\left.244-246{ }^{\circ} \mathrm{C}, \mathrm{ref}^{30}\right)$.

(5-Bromo-6-methyl-2,4-dioxo-1,2-dihydropyrimidin-3(4H)-yl)acetic acid (13). White powder, yield $0.92 \mathrm{~g}(70 \%), \mathrm{mp} 229-230{ }^{\circ} \mathrm{C}$; (mp 235-236 $\left.{ }^{\circ} \mathrm{C}, \mathrm{ref}^{31}\right)$.

General procedure for the synthesis of acetyl azides 1 and 16.

Method A. To a cooled $\left(0-5{ }^{\circ} \mathrm{C}\right)$ suspension of hydrazide 14 or $15(5 \mathrm{mmol})$, water $(15 \mathrm{~mL})$ and conc. hydrochloric acid (4 mL), a solution of sodium nitrite $(1.21 \mathrm{~g}, 17.5 \mathrm{mmol})$ in water $(5 \mathrm{~mL})$ was added dropwise under stirring. The reaction mixture was stirred at this temperature for 30 min., the precipitate was collected by filtration, washed with cold water and dried over sodium sulphate in vacuum.

(6-Methyl-2,4-dioxo-1,2-dihydropyrimidin-3(4H)-yl)acetyl azide (1). White solid, yield 0.76 g (73\%), mp $134-136{ }^{\circ} \mathrm{C}$; (mp135-137 ${ }^{\circ} \mathrm{C}$, $\left.\operatorname{ref}^{17}\right)$.

(5-Bromo-6-methyl-2,4-dioxo-1,2-dihydropyrimidin-3(4H)-yl)acetyl azide (16). White solid, yield $0.88 \mathrm{~g}(61 \%), \mathrm{mp} 248-250{ }^{\circ} \mathrm{C}$ (dec.). IR, $v, \mathrm{~cm}^{-1}: 1655,1709,1733(\mathrm{C}=\mathrm{O}), 2158\left(\mathrm{~N}_{3}\right)$, $3215(\mathrm{NH}) .{ }^{1} \mathrm{H}$ NMR $\left(\mathrm{CDCl}_{3}\right), \delta$, ppm: 2.40 (s, 3H, $\left.\mathrm{CH}_{3}\right), 4.75\left(\mathrm{~s}, 2 \mathrm{H}, \mathrm{NCH}_{2}\right), 10.48(\mathrm{~s}, 1 \mathrm{H}$, $\mathrm{NH}) ;{ }^{13} \mathrm{C}$ NMR $\left(\mathrm{CDCl}_{3}\right), \delta$, ppm: $20.3\left(\mathrm{CH}_{3}\right), 44.4\left(\mathrm{CH}_{2}\right), 96.8(5-\mathrm{C}), 149.0,151.5,158.7$ (2-C, 4-C, 6-C), $174.2(\mathrm{C}=\mathrm{O})$. HRMS (ES): $\left[\mathrm{MH}-\mathrm{CH}_{2} \mathrm{CON}_{3}\right]^{+}$calculated for $\mathrm{C}_{5} \mathrm{H}_{5} \mathrm{BrN}_{2} \mathrm{O}_{2}, 206.9600$; found 206.9600 .

Method B (only for azide 1). To a cooled to $-5{ }^{\circ} \mathrm{C}$ temperature suspension of acid $3(0.184 \mathrm{~g}, 1$ $\mathrm{mmol})$ in abs. acetone $(2 \mathrm{~mL})$, triethylamine $(0.28 \mathrm{~mL}, 2 \mathrm{mmol})$, then ethyl chloroformate $(0.19$ $\mathrm{mL}, 2 \mathrm{mmol})$ ) was added dropwise under stirring. The reaction mixture was stirred at this temperature for $30 \mathrm{~min}$, then a solution of sodium nitrite $(0.083 \mathrm{~g}, 1.2 \mathrm{mmol})$ in water $(0.5 \mathrm{~mL})$ was added dropwise under stirring. The reaction mixture was stirred at $-5-5{ }^{\circ} \mathrm{C}$ temperature for 7 $\mathrm{h}$ and was kept at temperature $5-10{ }^{\circ} \mathrm{C}$ overnight. Then the reaction mixture was diluted with water $(15 \mathrm{~mL})$, saturated with sodium chloride and extracted with dichloromethane $(3 \times 10 \mathrm{~mL})$. The extracts were combined, dried over sodium sulphate and filtered. After removal of the solvent $0.073 \mathrm{~g}(35 \%)$ of azide $1, \mathrm{mp} 135-137^{\circ} \mathrm{C}$ was obtained.

\section{General procedure for the synthesis of ureas 8, 17-23}

Method A. A mixture of azide 1 or $16(0.5 \mathrm{mmol})$ and corresponding aniline $(0.75 \mathrm{mmol})$ in dry benzene $(5 \mathrm{~mL})$ was heated at reflux for $2-4 \mathrm{~h}$. The reaction mixture was evaporated under reduced pressure to dryness. The residue was worked up with methanol to give a solid, which 
was collected by filtration, washed with methanol and crystallized from a mixture of dimethyl formamide and water.

$N$-(2,4-Dichlorophenyl)- $N^{\prime}$-[(6-methyl-2,4-dioxo-1,2-dihydropyrimidin-3(4H)yl)methyl]urea (8). White powder, yield $0.123 \mathrm{~g}(72 \%), \mathrm{mp} 221-223{ }^{\circ} \mathrm{C}$; (mp $223-225{ }^{\circ} \mathrm{C}$, $\left.\operatorname{ref}^{17}\right)$.

$N$-(2-Chlorophenyl)- $N^{\prime}$-[(6-methyl-2,4-dioxo-1,2-dihydropyrimidin-3(4H)-yl)methyl]urea (17). White powder, yield $0.099 \mathrm{~g}(64 \%), \mathrm{mp} 205-207^{\circ} \mathrm{C} . \mathrm{IR}, \mathrm{v}, \mathrm{cm}^{-1}$ : 1644, 1662, $1715(\mathrm{C}=\mathrm{O})$, 3293, $3346(\mathrm{NH}) .{ }^{1} \mathrm{H}$ NMR (DMSO-d $), \delta$, ppm: 2.05 (s, 3H, $\mathrm{CH}_{3}$ ), 5.23 (d, J $6.4 \mathrm{~Hz}, 2 \mathrm{H}$, $\left.\mathrm{NCH}_{2}\right), 5.51(\mathrm{~s}, 1 \mathrm{H}, 5-\mathrm{CH}), 6.98\left(\mathrm{t}, J 8.0 \mathrm{~Hz}, 1 \mathrm{H}, 4^{\prime}-\mathrm{ArH}\right), 7.25$ (t, J 8.0 Hz, 1H, 5'-ArH), 7.39 (d, $J 8.0 \mathrm{~Hz}, 1 \mathrm{H}, 3$ '-ArH), 7.77 (t, $\left.J 6.4 \mathrm{~Hz}, 1 \mathrm{H}, \mathrm{NCH}_{2} \mathrm{NH}\right), 8.06$ (d, J 8.0 Hz, 1H, 6'-ArH), 8.36 (s, $1 \mathrm{H}, \mathrm{CONH}), 11.21$ (br s, $1 \mathrm{H}, 1-\mathrm{NH}) ;{ }^{13} \mathrm{C} \mathrm{NMR}\left(\mathrm{DMSO}-d_{6}\right), \delta, \mathrm{ppm}: 18.8\left(\mathrm{CH}_{3}\right), 45.6\left(\mathrm{CH}_{2}\right)$, 99.0 (5-C), 122.0, 122.4, 123.8, 128.1, 129.9, 136.9 (Ar-C), 151.9, 153.1, 154.6, 163.6 (2-C, 4-C, 6-C, $\mathrm{C}=\mathrm{O}$ ). HRMS (ES): $[\mathrm{M}+\mathrm{Na}]^{+}$calculated for $\mathrm{C}_{13} \mathrm{H}_{13} \mathrm{ClN}_{4} \mathrm{NaO}_{3}, 331.0568$; found 331.0580 . $\boldsymbol{N}$-(4-Chlorophenyl)- $\boldsymbol{N}^{\prime}$-[(6-methyl-2,4-dioxo-1,2-dihydropyrimidin-3(4H)-yl)methyl]urea (18). White powder, yield $0.117 \mathrm{~g}(76 \%), \mathrm{mp} 245-247^{\circ} \mathrm{C} . \mathrm{IR}, \mathrm{v}, \mathrm{cm}^{-1}$ : 1642, 1682, $1732(\mathrm{C}=\mathrm{O})$, 3265, $3415(\mathrm{NH}) .{ }^{1} \mathrm{H}$ NMR (DMSO-d $)_{6}$ ), $\delta$, ppm: 2.04 (s, 3H, $\left.\mathrm{CH}_{3}\right), 5.21$ (d, J $6.4 \mathrm{~Hz}, 2 \mathrm{H}$, $\mathrm{NCH}_{2}$ ), 5.50 (s, 1H, 5-CH), 6.91 (t, J $\left.6.4 \mathrm{~Hz}, 1 \mathrm{H}, \mathrm{NCH}_{2} \underline{\mathrm{NH}}\right), 7.27$ (d, J $\left.8.8 \mathrm{~Hz}, 2 \mathrm{H}, 3^{\prime}, 5^{\prime}-\mathrm{ArH}\right)$, 7.38 (d, J $\left.8.8 \mathrm{~Hz}, 2 \mathrm{H}, 2^{\prime}, 66^{\prime}-\mathrm{ArH}\right), 8.90$ (s, 1H, CONH), 11.25 (br s, 1H, 1-NH); ${ }^{13} \mathrm{C}$ NMR (DMSO- $\left.d_{6}\right)$, $\delta$, ppm: $18.8\left(\mathrm{CH}_{3}\right), 45.7\left(\mathrm{CH}_{2}\right), 99.1$ (5-C), 119.9, 125.8, 129.3, 139.6 (Ar-C), $151.9,153.1,154.6,163.6(2-\mathrm{C}, 4-\mathrm{C}, 6-\mathrm{C}, \mathrm{C}=\mathrm{O})$. HRMS (ES): $[\mathrm{M}+\mathrm{Na}]^{+}$calculated for $\mathrm{C}_{13} \mathrm{H}_{13} \mathrm{ClN}_{4} \mathrm{NaO}_{3}, 331.0568$; found 331.0579.

$\boldsymbol{N}$-(2,6-Dichlorophenyl)- $\boldsymbol{N}^{\prime}$-[(6-methyl-2,4-dioxo-1,2-dihydropyrimidin-3(4H)-

yl)methyl]urea (19). White powder, yield $0.091 \mathrm{~g}(53 \%), \mathrm{mp} \mathrm{224-226}{ }^{\circ} \mathrm{C} . \mathrm{IR}, \mathrm{v}, \mathrm{cm}^{-1}$ : 1648 , 1660, $1724(\mathrm{C}=\mathrm{O}), 3249,3351(\mathrm{NH}) .{ }^{1} \mathrm{H}$ NMR (DMSO-d $), \delta$, ppm: $2.06\left(\mathrm{~s}, 3 \mathrm{H}, \mathrm{CH}_{3}\right), 5.21(\mathrm{~d}, J$ $\left.6.4 \mathrm{~Hz}, 2 \mathrm{H}, \mathrm{NCH}_{2}\right), 5.51(\mathrm{~s}, 1 \mathrm{H}, 5-\mathrm{CH}), 7.08\left(\mathrm{t}, J 6.4 \mathrm{~Hz}, 1 \mathrm{H}, \mathrm{NCH}_{2} \mathrm{NH}\right), 7.27$ (t, J 8.0 Hz, 1H, 4'-ArH), 7.48 (d, J $8.0 \mathrm{~Hz}, 2 \mathrm{H}, 3$ ', $\left.5^{\prime}-\mathrm{ArH}\right), 8.31$ (s, 1H, CONH), 11.21 (s, 1H, 1-NH); ${ }^{13} \mathrm{C}$ NMR (DMSO- $\left.d_{6}\right)$, $\delta$, ppm: $18.6\left(\mathrm{CH}_{3}\right), 46.0\left(\mathrm{CH}_{2}\right), 98.8$ (5-C), 128.5, 128.8, 134.0 (2) (Ar-C), 151.7, 152.7, 154.3, $163.2(2-\mathrm{C}, 4-\mathrm{C}, 6-\mathrm{C}, \mathrm{C}=\mathrm{O})$. HRMS (ES): $[\mathrm{M}+\mathrm{H}]^{+}$calculated for $\mathrm{C}_{13} \mathrm{H}_{12} \mathrm{Cl}_{2} \mathrm{~N}_{4} \mathrm{O}_{3}$, 343.0359 ; found 343.0361 .

$\boldsymbol{N}$-(4-Bromophenyl)- $\boldsymbol{N}^{\prime}$-[(6-methyl-2,4-dioxo-1,2-dihydropyrimidin-3(4H)-yl)methyl]urea (20). White powder, yield $0.115 \mathrm{~g}(65 \%), \mathrm{mp} 240-242{ }^{\circ} \mathrm{C} . \mathrm{IR}, \mathrm{v}, \mathrm{cm}^{-1}$ : 1643, $1733(\mathrm{C}=\mathrm{O}), 3266$, $3414(\mathrm{NH}) .{ }^{1} \mathrm{H}$ NMR (DMSO-d $)$ ), $\delta$, ppm: 2.04 (s, 3H, $\left.\mathrm{CH}_{3}\right), 5.20$ (d, J $\left.6.4 \mathrm{~Hz}, 2 \mathrm{H}, \mathrm{NCH}_{2}\right), 5.49$ (s, 1H, 5-CH), 6.90 (t, J 6.4 Hz, 1H, $\mathrm{NCH}_{2} \underline{\mathrm{NH}}$ ), 7.32 (d, J $8.8 \mathrm{~Hz}, 2 \mathrm{H}, 3$ ',5'-ArH), 7.39 (d, J 8.8 $\left.\mathrm{Hz}, 2 \mathrm{H}, 2^{\prime}, 6^{\prime}-\mathrm{ArH}\right), 8.89$ (s, 1H, CONH), 11.17 (br s, 1H, 1-NH); ${ }^{13} \mathrm{C}$ NMR (DMSO- $\left.d_{6}\right), \delta$, ppm: $18.8\left(\mathrm{CH}_{3}\right), 45.8\left(\mathrm{CH}_{2}\right), 99.1$ (5-C), 113.7, 120.4, 132.2, 139.9 (Ar-C), 151.9, 153.2, 154.6, 163.6 (2-C, 4-C, 6-C, C=O). HRMS (ES): $[\mathrm{M}+\mathrm{H}]^{+}$calculated for $\mathrm{C}_{13} \mathrm{H}_{13} \mathrm{BrN}_{4} \mathrm{O}_{3}, 353.0244$; found 353.0255 .

$\boldsymbol{N}$-(2,4-Dibromophenyl)- $\boldsymbol{N}^{\prime}$-[(6-methyl-2,4-dioxo-1,2-dihydropyrimidin-3(4H)-

yl)methyl]urea (21). White powder, yield $0.156 \mathrm{~g}(72 \%), \mathrm{mp} 236-237{ }^{\circ} \mathrm{C}$. IR, $\mathrm{v}, \mathrm{cm}^{-1}: 1656$, $1718(\mathrm{C}=\mathrm{O}), 3327(\mathrm{NH}) .{ }^{1} \mathrm{H}$ NMR $\left(\mathrm{DMSO}-d_{6}\right), \delta$, ppm: $2.05\left(\mathrm{~s}, 3 \mathrm{H}, \mathrm{CH}_{3}\right), 5.22(\mathrm{~d}, J 6.0 \mathrm{~Hz}, 2 \mathrm{H}$, 
$\mathrm{NCH}_{2}$ ), 5.50 (s, 1H, 5-CH), 7.47 (d, J 8.8 Hz, 1H, 5'-ArH), 7.77 (s, 1H, 3'-ArH), 7.89 (t, J 6.0 $\mathrm{Hz}, 1 \mathrm{H}, \mathrm{NCH}_{2} \mathrm{NH}$ ), 8.01 (d, J $\left.8.8 \mathrm{~Hz}, 1 \mathrm{H}, 6^{\prime}-\mathrm{ArH}\right), 8.25$ (s, 1H, CONH), 11.19 (br s, 1H, 1-NH); ${ }^{13} \mathrm{C}$ NMR (DMSO- $\left.d_{6}\right), \delta$, ppm: $18.6\left(\mathrm{CH}_{3}\right), 45.3\left(\mathrm{CH}_{2}\right), 98.8(5-\mathrm{C}), 113.5,114.2,123.6,131.2$, 134.5, 137.7 (Ar-C), 151.6, 152.7, 154.2, 163.0 (2-C, 4-C, 6-C, C=O. HRMS (ES): [M+H] calculated for $\mathrm{C}_{13} \mathrm{H}_{12} \mathrm{Br}_{2} \mathrm{~N}_{4} \mathrm{O}_{3}, 432.9329$; found 432.9340 .

$\mathrm{N}$-[(5-Bromo-6-methyl-2,4-dioxo-1,2-dihydropyrimidin-3(4H)-yl)methyl]- $\mathrm{N}^{\prime}$-(2,4dichlorophenyl)urea (22). White powder, yield $0.15 \mathrm{~g}(71 \%), \mathrm{mp} 247-249{ }^{\circ} \mathrm{C} . \mathrm{IR}, \mathrm{v}, \mathrm{cm}^{-1}$ : 1659, $1717(\mathrm{C}=\mathrm{O}), 3319(\mathrm{NH}) .{ }^{1} \mathrm{H}$ NMR (DMSO-d $), \delta$, ppm: $2.24\left(\mathrm{~s}, 3 \mathrm{H}, \mathrm{CH}_{3}\right), 5.28(\mathrm{~d}, J 6.4$ $\mathrm{Hz}, 2 \mathrm{H}, \mathrm{NCH}_{2}$ ), 7.32 (d, J $9.2 \mathrm{~Hz}, 1 \mathrm{H}, 5$ '-ArH), 7.54 (s, 1H, 3'-ArH), 7.93 (t, J $6.4 \mathrm{~Hz}, 1 \mathrm{H}$, $\mathrm{NCH}_{2} \underline{\mathrm{NH}}$ ), 8.16 (d, J 9.2 Hz, 1H, 6'-ArH), 8.44 (s, 1H, CONH), 11.67 (s, 1H, 1-NH); ${ }^{13} \mathrm{C}$ NMR (DMSO- $\left.d_{6}\right), \delta$, ppm: $20.0\left(\mathrm{CH}_{3}\right), 46.8\left(\mathrm{CH}_{2}\right), 95.0$ (5-C), 122.4, 122.6, 126.1, 127.9, 128.9, 136.1 (Ar-C), 150.3, 151.3, 154.2, $159.4(2-\mathrm{C}, 4-\mathrm{C}, 6-\mathrm{C}, \mathrm{C}=\mathrm{O})$. HRMS (ES): $[\mathrm{M}+\mathrm{H}]^{+}$calculated for $\mathrm{C}_{13} \mathrm{H}_{11} \mathrm{BrCl}_{2} \mathrm{~N}_{4} \mathrm{O}_{3}, 422.9441$; found 422.9439 .

$\mathrm{N}$-[(5-Bromo-6-methyl-2,4-dioxo-1,2-dihydropyrimidin-3(4H)-yl)methyl]- $\boldsymbol{N}^{\prime}$-(2,4-

dibromophenyl)urea (23). White powder, yield $0.176 \mathrm{~g}(69 \%), \mathrm{mp} 247-248{ }^{\circ} \mathrm{C} . \mathrm{IR}, \mathrm{v}, \mathrm{cm}^{-1}$ : 1661, $1718(\mathrm{C}=\mathrm{O}), 3325(\mathrm{NH}) .{ }^{1} \mathrm{H}$ NMR (DMSO-d $), \delta$, ppm: 2.24 (s, 3H, $\left.\mathrm{CH}_{3}\right), 5.28(\mathrm{~d}, J 4.8$ $\left.\mathrm{Hz}, 2 \mathrm{H}, \mathrm{NCH}_{2}\right), 7.47$ (d, J $\left.8.4 \mathrm{~Hz}, 1 \mathrm{H}, 5^{\prime}-\mathrm{ArH}\right), 7.78$ (s, 1H, 3'-ArH), 8.0 (br s, 1H, $\mathrm{NCH}_{2} \underline{\mathrm{NH}}$ ), 8.02 (br s, 1H, 6'-ArH), 8.26 (s, 1H, CONH), 11.67 (s, 1H, 1-NH); ${ }^{13} \mathrm{C}$ NMR (DMSO- $\left.d_{6}\right), \delta$, ppm: $20.0\left(\mathrm{CH}_{3}\right), 46.8\left(\mathrm{CH}_{2}\right), 95.0$ (5-C), 113.5, 114.2, 123.5, 131.2, 134.5, 137.6 (Ar-C), 150.3, 151.3, 154.2, 159.4 (2-C, 4-C, 6-C, $C=\mathrm{O})$. HRMS (ES): $[\mathrm{M}+\mathrm{H}]^{+}$calculated for $\mathrm{C}_{13} \mathrm{H}_{11} \mathrm{Br}_{3} \mathrm{~N}_{4} \mathrm{O}_{3}$, 510.8434 ; found 510.8440 .

Method B. To a stirred mixture of acid 12 or $13(0.5 \mathrm{mmol})$ in dry benzene $(5 \mathrm{~mL})$ triethylamine $(0.28 \mathrm{~mL}, 2 \mathrm{mmol})$ was added dropwise. The reaction mixture was stirred at room temperature for 10 min., then diphenyl phosphoryl azide $(0.44 \mathrm{~mL}, 2 \mathrm{mmol})$ was added dropwise. The reaction mixture was stirred at room temperature for $24 \mathrm{~h}$, then the corresponding aniline $(0.75$ mmol) was added. The reaction mixture was heated at reflux for $3 \mathrm{~h}$ and evaporated under reduced pressure to dryness. The residue was worked up with methanol to give a solid, which was collected by filtration, washed with methanol and dried to yield $0.067 \mathrm{~g} \mathrm{(39 \% )} \mathrm{of} \mathbf{8}, 0.014 \mathrm{~g}$ (8\%) of 19, $0.11 \mathrm{~g}(52 \%)$ of $21,0.072 \mathrm{~g} \mathrm{(34 \% )}$ of 22 or $0.061 \mathrm{~g}(24 \%)$ of 23.

General procedure for the synthesis of hydrazones 24 and 25. A mixture of hydrazide 14 $(0.099 \mathrm{~g}, 0.5 \mathrm{mmol})$ and 2-propanol $(10 \mathrm{~mL})$ was heated at reflux for $3 \mathrm{~min}$., then cooled to $70-$ $75{ }^{\circ} \mathrm{C}$ temperature and the corresponding benzaldehyde was added. The reaction mixture was cooled to room temperature, the resultant precipitate was collected by filtration, washed with methanol and dried.

$N^{\prime}$-[(2-Chlorophenyl)methylene]-(6-methyl-2,4-dioxo-1,2-dihydropyrimidin-3(4H)yl)acetohydrazide (24). White solid, yield $0.138 \mathrm{~g}(86 \%), \mathrm{mp} 311-312{ }^{\circ} \mathrm{C}$. IR, $\mathrm{v}, \mathrm{cm}^{-1}: 1657$, 1682, $1714(\mathrm{C}=\mathrm{O}), 3189,3233,3436(\mathrm{NH}) .{ }^{1} \mathrm{H}$ NMR (DMSO-d $), \delta$, ppm: $2.09\left(\mathrm{~s}, 3 \mathrm{H}, \mathrm{CH}_{3}\right)$, 4.47, $4.88\left(2 \mathrm{~s}, 2 \mathrm{H}, \mathrm{NCH}_{2}\right), 5.54(\mathrm{~s}, 1 \mathrm{H}, 5-\mathrm{CH}), 7.40-7.47$ (m, 2H, 4',5'-ArH), 7.53 (d, J 7.2 Hz, 1H, 3'-ArH), 7.93, 8.01 (2d, J 7.2 Hz, 1H, 6'-ArH), 8.40, 8.58 (2s, 1H, N=CH), 11.24, 11.27 (2s, $1 \mathrm{H}, \mathrm{CONH}), 11.81,11.89(2 \mathrm{~s}, 1 \mathrm{H}, 1-\mathrm{NH}) ;{ }^{13} \mathrm{C} \mathrm{NMR}\left(\mathrm{DMSO}-d_{6}\right), \delta, \mathrm{ppm}: 18.6\left(\mathrm{CH}_{3}\right), 41.2$ 
$\left(\mathrm{CH}_{2}\right)$, 98.6 (5-C), 127.3, 128.1, 130.4, 131.7, 131.8, 133.4 (Ar-C), 140.4, 151.8, 152.2, 163.1, 168.7 (2-C, 4-C, 6-C, $\mathrm{N}=\mathrm{CH}, \mathrm{C}=\mathrm{O}$ ). HRMS (ES): $[\mathrm{M}+\mathrm{H}]^{+}$calculated for $\mathrm{C}_{14} \mathrm{H}_{13} \mathrm{ClN}_{4} \mathrm{O}_{3}$, 321.0758; found 321.0749 .

$\boldsymbol{N}^{\prime}$-[(2,4-Dichlorophenyl)methylene]-(6-methyl-2,4-dioxo-1,2-dihydropyrimidin-3(4H)yl)acetohydrazide (25). White solid, yield $0.137 \mathrm{~g}(77 \%), \mathrm{mp} 318-320{ }^{\circ} \mathrm{C}$. IR, $\mathrm{v}, \mathrm{cm}^{-1}$ : 1652 , 1660, $1714(\mathrm{C}=\mathrm{O}), 3190,3436(\mathrm{NH}) .{ }^{1} \mathrm{H}$ NMR (DMSO-d $), \delta$, ppm: 2.08 (s, 3H, $\left.\mathrm{CH}_{3}\right), 4.47$, $4.87\left(2 \mathrm{~s}, 2 \mathrm{H}, \mathrm{NCH}_{2}\right), 5.53(\mathrm{~s}, 1 \mathrm{H}, 5-\mathrm{CH}), 7.49$ (d, J $\left.8.4 \mathrm{~Hz}, 1 \mathrm{H}, 5^{\prime}-\mathrm{ArH}\right), 7.72$ (s, 1H, 3'-ArH), 7.93, 8.02 (2d, $J 8.4 \mathrm{~Hz}, 1 \mathrm{H}, 6$ '-ArH), 8.34, $8.53(2 \mathrm{~s}, 1 \mathrm{H}, \mathrm{N}=\mathrm{CH}), 11.25,11.27$ (2s, 1H, CONH), 11.86, $11.94(2 \mathrm{~s}, 1 \mathrm{H}, 1-\mathrm{NH}) ;{ }^{13} \mathrm{C}$ NMR (DMSO-d $), \delta$, ppm: $18.6\left(\mathrm{CH}_{3}\right), 41.2\left(\mathrm{CH}_{2}\right), 98.6(5-\mathrm{C})$, 128.4, 128.6, 129.8, 130.8, 134.1, 135.4 (Ar-C), 139.3, 151.8, 152.2, 163.0, 168.8 (2-C, 4-C, 6$\mathrm{C}, \mathrm{N}=\mathrm{CH}, \mathrm{C}=\mathrm{O}$ ). HRMS (ES): $[\mathrm{M}+\mathrm{Na}]^{+}$calculated for $\mathrm{C}_{14} \mathrm{H}_{12} \mathrm{Cl}_{2} \mathrm{~N}_{4} \mathrm{NaO}_{3}, 377.0179$; found 377.0171 .

$\mathrm{N}$-[(6-Methyl-2,4-dioxo-1,2-dihydropyrimidin-3(4H)-yl)methyl]hydrazinecarboxamide (26). A mixture of azide $1(0.209 \mathrm{~g}, 1 \mathrm{mmol})$ and dry benzene $(20 \mathrm{~mL})$ was heated at reflux for $2 \mathrm{~h}$, then the solvent was removed under reduced pressure. The residue was dissolved in dry diethyl ether $(40 \mathrm{~mL})$ and $100 \%$ hydrazine hydrate $(0.06 \mathrm{~mL}, 1.2 \mathrm{mmol})$ was added. The reaction mixture was kept at room temperature for $1 \mathrm{~h}$, the resultant precipitate was collected by filtration, washed with methanol and dried to yield $0.141 \mathrm{~g}(66 \%)$ of compound $26, \mathrm{mp} 198-200{ }^{\circ} \mathrm{C}$. IR, $v$, $\mathrm{cm}^{-1}$ : 1644, 1683, $1734(\mathrm{C}=\mathrm{O}) ; 3223,3364(\mathrm{NH}) .{ }^{1} \mathrm{H}$ NMR (DMSO-d $)$ ), $\delta$, ppm: 2.03 (s, 3H, $\mathrm{CH}_{3}$ ), 4.19 (br s, 2H, $\mathrm{NH}_{2}$ ), 5.16 (d, J $6.8 \mathrm{~Hz}, 2 \mathrm{H}, \mathrm{NCH}_{2}$ ), 5.46 (s, 1H, 5-CH), 6.96 (br s, 1H, $\mathrm{NCH}_{2} \underline{\mathrm{NH}}$ ), 7.23 (s, 1H, CONH), 10.75 (br s, $\left.1 \mathrm{H}, \mathrm{NH}\right) ;{ }^{13} \mathrm{C} \mathrm{NMR}$ (DMSO-d $)_{6}$ ), $\delta$, ppm: 18.6 $\left(\mathrm{CH}_{3}\right), 45.5\left(\mathrm{CH}_{2}\right), 98.8$ (5-C), 151.7, 152.4, 159.1, 163.1 (2-C, 4-C, 6-C, C=O). HRMS (ES): $[\mathrm{M}+\mathrm{H}]^{+}$calculated for $\mathrm{C}_{7} \mathrm{H}_{11} \mathrm{~N}_{5} \mathrm{O}_{3}, 214.0935$; found 214.0929 .

General procedure for the synthesis of semicarbazones 27 and 28. A mixture of semicarbazide $26(0.107 \mathrm{~g}, 0.5 \mathrm{mmol})$, the corresponding benzaldehyde $(0.6 \mathrm{mmol})$ and abs. ethanol $(25 \mathrm{~mL})$ was stirred at room temperature for $24 \mathrm{~h}$. Then the solid formed was collected by filtration, washed with ethanol and dried.

(2-Chlorobenzylidene]- $N$-[(6-methyl-2,4-dioxo-1,2-dihydropyrimidin-3(4H)-

yl)methyl]hydrazinecarboxamide (27). White solid, yield $0.086 \mathrm{~g}(51 \%), \mathrm{mp} 241-242{ }^{\circ} \mathrm{C}$. IR, $v, \mathrm{~cm}^{-1}:$ 1642, 1687, $1714(\mathrm{C}=\mathrm{O}), 3211,3341,3425(\mathrm{NH}) .{ }^{1} \mathrm{H}$ NMR (DMSO-d $), \delta, \mathrm{ppm}: 2.04$ (s, $\left.3 \mathrm{H}, \mathrm{CH}_{3}\right), 5.29$ (d, J $\left.6.4 \mathrm{~Hz}, 2 \mathrm{H}, \mathrm{NCH}_{2}\right), 5.49$ (s, 1H, 5-CH), 7.32 (t, J $6.4 \mathrm{~Hz}, 1 \mathrm{H}, \mathrm{NCH}_{2} \mathrm{NH}$ ), 7.39-7.41 (m, 2H, 4',5'-ArH), 7.49 (d, J $6.0 \mathrm{~Hz}, 1 \mathrm{H}, 3^{\prime}-\mathrm{ArH}$ ), 7.93 (d, J $6.0 \mathrm{~Hz}, 1 \mathrm{H}, 6$ '-ArH), $8.25(\mathrm{~s}, 1 \mathrm{H}, \mathrm{N}=\mathrm{CH}), 10.83(\mathrm{~s}, 1 \mathrm{H}, \mathrm{CONH}), 11.19(\mathrm{~s}, 1 \mathrm{H}, 1-\mathrm{NH}) ;{ }^{13} \mathrm{C}$ NMR (DMSO- $\left.d_{6}\right), \delta, \mathrm{ppm}$ : $18.6\left(\mathrm{CH}_{3}\right), 45.6\left(\mathrm{CH}_{2}\right), 98.9$ (5-C), 127.0, 127.9, 130.4, 131.2, 132.0, 132.9 (Ar-C), 136.9, 151.7, 152.6, 154.5, $163.1(2-\mathrm{C}, 4-\mathrm{C}, 6-\mathrm{C}, \mathrm{N}=\mathrm{CH}, \mathrm{C}=\mathrm{O})$. HRMS (ES): $[\mathrm{M}+\mathrm{H}]^{+}$calculated for $\mathrm{C}_{14} \mathrm{H}_{14} \mathrm{ClN}_{5} \mathrm{O}_{3}, 336.0858$; found 336.0868 .

(2,4-Dichlorobenzylidene)- $N$-[(6-methyl-2,4-dioxo-1,2-dihydropyrimidin-3(4H)-

yl)methyl]hydrazinecarboxamide (28). White solid, yield $0.126 \mathrm{~g} \mathrm{(68 \% ),} \mathrm{mp} 255-260{ }^{\circ} \mathrm{C}$. IR, $v, \mathrm{~cm}^{-1}: 1645,1714(\mathrm{C}=\mathrm{O}), 3212,3359,3407(\mathrm{NH}) .{ }^{1} \mathrm{H}$ NMR (DMSO-d $), \delta$, ppm: 2.04 (s, 3H, 
$\left.\mathrm{CH}_{3}\right), 5.29$ (s, 2H, $\left.\mathrm{NCH}_{2}\right), 5.48(\mathrm{~s}, 1 \mathrm{H}, 5-\mathrm{CH}), 7.31$ (s, 1H, $\left.\mathrm{NCH}_{2} \underline{\mathrm{NH}}\right), 7.49$ (d, J $6.8 \mathrm{~Hz}, 1 \mathrm{H}, 5^{\prime}-$ ArH), 7.67 (s, 1H, 3'-ArH), 7.95 (d, J 6.8 Hz, 1H, 6'-ArH), 8.20 (s, 1H, N=CH), 10.84 (s, 1H, $\mathrm{CONH}), 11.09(\mathrm{~s}, 1 \mathrm{H}, 1-\mathrm{NH}) ;{ }^{13} \mathrm{C} \mathrm{NMR}\left(\mathrm{DMSO}-d_{6}\right), \delta, \mathrm{ppm}: 18.6\left(\mathrm{CH}_{3}\right), 45.6\left(\mathrm{CH}_{2}\right), 98.9$ (5C), 128.2, 128.3, 129.8, 131.2, 133.6, 134.7 (Ar-C), 135.9, 151.7, 152.4, 154.5, 163.1 (2-C, 4-C, 6-C, $\mathrm{N}=\mathrm{CH}, \mathrm{C}=\mathrm{O}$ ). HRMS (ES): $[\mathrm{M}+\mathrm{H}]^{+}$calculated for $\mathrm{C}_{14} \mathrm{H}_{13} \mathrm{Cl}_{2} \mathrm{~N}_{5} \mathrm{O}_{3}, 370.0468$; found 370.0479 .

\section{References}

1. Keche, A. P.; Hatnapure, G. D.; Tale, R. H.; Rodge, A. H.; Birajdar, S. S.; Kamble, V. M. Bioorg. Med. Chem. Letters 2012, 22, 3445. http://dx.doi.org/10.1016/j.bmcl.2012.03.092

2. Novikov, M. S.; Valuev-Elliston, V. T.; Babkov, D. A.; Paramonova, M. P.; Ivanov, A. V.; Gavryushov, S. A.; Khandazhinskaya, A. L.; Kochetkow, S. N.; Pannecouque, C.; Andrei, G.; Snoeck, R.; Balzarini, J.; Seley-Radtke, K. L. Bioorg. Med. Chem. 2013, 21, 1150. http://dx.doi.org/10.1016/j.bmc.2012.12.027

3. Pan, L.; Jiang, Y.; Liu, Z.; Liu, X.-H.; Liu, Z.; Wang G.; Li, Z.-M.; Wang, D. Eur. J. Med. Chem. 2012, 50, 18. http://dx.doi.org/10.1016/j.ejmech.2012.01.011

4. Grillot, A.-L.; Le Tiran, A.; Shannon, D.; Krueger, E.; Liao, Y.; O’Dowd., H.; Tang, Q.; Ronkin, S.; Wang, T.; Waal, N.; Li, P.; Lauffer, D.; Sizensky, E.; Tanoury, J.; Perola, E.; Grossman, T. H.; Doyle, T.; Hanzelka, B.; Jones, S.; Dixit V.; Ewing, N.; Liao, S.; Boucher, B.; Jacobs, M.; Bennani, Y.; Charifson, P. S. J. Med. Chem. 2014, 57, 8792. http://dx.doi.org/10.1021/jm500563g

5. Tale, R. H.; Rodge, A. H.; Hatnapure, G. D.; Keche, A. P. Bioorg. Med. Chem. Letters 2011, 21,4648 .

http://dx.doi.org/10.1016/j.bmcl.2011.03.062

6. Ding, H. X.; Leverett, C. A.; Kyne (Jr.), R. E.; Lui, K. K.-C.; Fink, S. J.; Flick, A. C.; O'Donnell, C. J. Bioorg. Med. Chem. 2015, 23, 1895.

http://dx.doi.org/10.1016/j.bmc.2015.02.056

7. Saurat, T.; Buron, F.; Rodriques, N.; de Tauzia, M. L.; Colliandre, L.; Bourg, S.; Bonnet, P.; Guillaumet, G.; Akssira, M.; Corlu, A.; Guillouzo, C,; Berthier, P.; Rio, P.; Jourdan, M.-L.; Benedetti, H.; Routier, S. J. Med. Chem. 2014, 57, 613. http://dx.doi.org/10.1021/jm401138v

8. Duan, Y. C.; Zheng, Y. C.; Li, X. C.; Wang, M. M.; Ye, X. W.; Guan, Y. Y.; Liu, G. Z.; Zheng, J. X.; Liu, H. M. Eur. J. Med. Chem. 2013, 64, 99.

http://dx.doi.org/10.1016/j.ejmech.2013.03.058

9. Wang, A. Y.; Lu, Y,; Zhu, H. L.; Jiao, Q. C. Oncol. Lett. 2012, 3, 373. http://dx.doi.org/10.3892/ol.2011.474 
10. Chimenti, F.; Bizzarri, B.; Bolasco, A.; Secci, D.; Chimenti, P.; Carradori, S.; Granese, A.; Rivanera, D.; Frishberg, N.; Bordon, C.; Jones-Brando, L. J. Med. Chem. 2009, 52, 4574. http://dx.doi.org/10.1021/jm9005862

11. Basarab, G. S.; Manchester, J. I.; Bist, S.; Boriack-Sjodin, P. A.; Dangel, B.; Illingworth, R.; Sherer, B. A.; Sriram, S.; Uria-Nickelsen, M.; Eakin, A. E. J. Med. Chem. 2013, 56, 8712. http://dx.doi.org/10.1021/jm401208b

12. Boechat, N.; Ferreira, V., F.; Ferreira, S. B.; Ferreira, M. L. G.; da Silva, F. C.; Bastos, M. M.; Costa, M. S.; Lourenco, M. C. S.; Pinto, A. C.; Krettli, A. U.; Aguiar, A. C.; Teixeira, B. M.; da Silva, N. V.; Martins, P. R. C.; Bezerra, F. A. F. M.; Camilo, A. L. S.; da Silva, G., P.; Costa, C. C. P. J. Med. Chem. 2011, 54, 5988.

http://dx.doi.org/10.1021/jm2003624

13. Keche, A. P.; Hatnapure, G. D.; Tale, R. H.; Rodge, A. H.; Kamble, V. M. Bioorg. Med. Chem. Letters 2012, 22, 6611.

http://dx.doi.org/10.1016/j.bmcl.2012.08.118

14. Yang, W.; Wang, Y.; Lai, A.; Qiao, J. X.; Wang, T. C.; Hua, J.; Price, L. A.; Shen, H.; Chen, X.; Wong, P.; Crain, E.; Watson, C.; Huang, C. S.; Seiffert, D. A.; Rehfuss, R.; Wexler, R. R.; Lam, P. Y. S. J. Med. Chem. 2014, 57, 6150.

http://dx.doi.org/10.1021/jm5006226

15. Voight, E. A.; Gomtsyan, A. R.; Daanen, J. F.; Perner, R. J.; Schmidt, R. G.; Bayburt, E. K.; DiDomenico, S.; McDonald, H. A.; Puttfarchen, P. S.; Chen, J.; Neelands, T. R.; Bianchi, B. R.; Han, P.; Reilly, R. M.; Franklin, P. H.; Segreti, J. A.; Nelson, R. A.; Su, Z.; King, A. J.; Polakowski, J. S.; Baker, S. J.; Gauvin, D. M.; Lewis, L. R.; Mikusa, J. P.; Joshi, S. K.; Faltynek C. R.; Kym, P. R.; Kort, M. E. J. Med. Chem. 2014, 57, 7412.

http://dx.doi.org/10.1021/jm500916t

16. Rineh, A.; Mahmoodi, N.; Abdollahi, M.; Foroumadi, A.; Sorkhi, M.; Shafiee, A. Arch. Pharm. Chem. Life Sci. 2007, 340, 409. http://dx.doi.org/10.1002/ardp.200700045

17. Jakubkiene, V.; Kacnova, Z.; Burbuliene, M. M.; Vainilavicius, P. Arkivoc 2010, (xi), 39.

18. Cabrera-Benitez, N. E.; Perez-Roth, E.; Casula, M.; Ramos-Nuez, A.; Rios-Luci, C.; Rodriguez-Gallego, C.; Sologuren, I.; Jakubkiene, V.; Slutsky, A. S.; Padron, J. M.; Villar, J. PLOS ONE 2012, 7, e48468.

http://dx.doi.org/10.1371/journal.pone.0048468

19. Jakubkiene, V.; Burbuliene, M. M.; Udrenaite, E.; Garaliene V.; Vainilavicius, P. Pharmazie 2002, 57, 610 .

20. Vainilavicius, P.; Smicius, R.; Jakubkiene, V.; Tumkevicius, S. Monatsh. Chem. 2001, 132, 825.

21. Zanirato, P. Arkivoc 2009, (i), 97.

22. Dogan, S. D.; Demirpolat, E.; Aycan, M .B. Y.; Balci, M. Tetrahedron 2015, 71, 252. http://dx.doi.org/10.1016/j.tet.2014.11.057

23. Ergun, M.; Dengiz, C.; Ozer, M. S.; Sahin, E.; Balci, M.; Tetrahedron 2014, 70, 5993. 
24. Danielli, F.; Zanirato, P. Arkivoc 2000, (i), 58.

25. Fidesser, E.; Haider, N.; Jbara, R. Arkivoc 2001, (v), 133.

26. Rumi, L.; Pfleger, C.; Spurr, P.; Klinkhammer, U.; Bannwarth. Org. Process Res. Dev. 2009, $13,747$.

http://dx.doi.org/10.1021/op9000516

27. Koza, G.; Ozcan, Z.; Sahin, E.; Balci, M. Tetrahedron 2009, 65, 5973.

http://dx.doi.org/10.1016/j.tet.2009.05.090

28. Kuodis, Z.; Rutavičius, A.; Matijoška, A.; Eicher-Lorka, O. Cent. Eur. J. Chem. 2007, 5, 996.

http://dx.doi.org/10.2478/s11532-007-0043-7

29. Palla, G.; Predieri, G.; Domiano, P.; Vignali, C.; Turner, W. Tetrahedron 1986, 42, 3649. http://dx.doi.org/10.1016/S0040-4020(01)87332-4

30. Vainilavicius, P.; Sedereviciute, V. Khim. Geterotsikl. Soedin. 1987, 1520.

31. Jakubkiene, V.; Kacnova, Z.; Burbuliene, M. M.; Vainilavicius, P. J. Heterocyclic Chem. 2008, 45, 1391.

http://dx.doi.org/10.1002/jhet.5570450522 